\title{
Combined effects of hypertension and heart rate on the risk of stroke and coronary heart disease: a population-based prospective cohort study among Inner Mongolians in China
}

\author{
Chongke Zhong ${ }^{1,2,5}$, Xiaoyan Zhong ${ }^{3,5}$, Tian $\mathrm{Xu}^{1}$, Hao Peng ${ }^{1}$, Hongmei $\mathrm{Li}^{1}$, Mingzhi Zhang ${ }^{1}$, Aili Wang ${ }^{1}$, \\ Tan $\mathrm{Xu}^{1}$, Yingxian $\mathrm{Sun}^{4}$ and Yonghong Zhang ${ }^{1,2}$
}

The purpose of this study was to prospectively evaluate the combined effects of hypertension and heart rate on the risk of stroke and coronary heart disease (CHD) among Inner Mongolians. Based a cross-sectional survey in 2002-2003, a prospective cohort study was conducted among 2530 Mongolian people. We categorized the participants into four subgroups according to blood pressure and heart rate. Cox proportional hazards models and receiver operating characteristic (ROC) curves were used to evaluate the association between hypertension, heart rate, and stroke and CHD incidence. During the follow-up period, a total of 120 stroke and $75 \mathrm{CHD}$ patients were observed. Compared with normotensives with a heart rate $<80$ b.p.m., in a multivariateadjusted model, the hazard ratios (HRs; $95 \%$ confidence intervals $(\mathrm{Cls})$ ) of stroke for hypertensives with a heart rate $<80$ b.p.m. and hypertensives with heart rate $\geqslant 80$ b.p.m. were $3.21(1.80-5.70)$ and $3.59(1.95-6.62$ ), respectively (all $P<0.05$ ); the HRs $(95 \% \mathrm{Cl})$ of $\mathrm{CHD}$ for hypertensives with a heart rate $<80$ b.p.m. and hypertensives with a heart rate $\geqslant 80$ b.p.m. were 2.09 (1.07-4.07) and $2.76(1.43-5.36$ ), respectively (all $P<0.05$ ). For both stroke and CHD incidence, the area under ROC curve for a model containing hypertension and a heart rate $\geqslant 80$ b.p.m. along with other conventional factors was significantly larger than the one containing only other conventional factors (all $P<0.05$ ). Hypertensives with a high heart rate had the highest risk of stroke and CHD among the Inner Mongolians. These results indicate that the coexistence of hypertension and high heart rate may be a valuable predictor of stroke and CHD incidence.

Hypertension Research (2015) 38, 883-888; doi:10.1038/hr.2015.90; published online 20 August 2015

Keywords: cohort study; coronary heart disease; heart rate; stroke

\section{INTRODUCTION}

Presently, cardiovascular disease (CVD) has become a major public health issue worldwide, ${ }^{1-3}$ and the burden of CVD is high and growing. Deaths from CVD are estimated to rise to 23.4 million in 2030, an $\sim 37 \%$ increase from 2004. Furthermore, the leading causes of death in the world in 2030 are predicted to be ischemic heart disease and stroke, both are major components of CVD. ${ }^{4}$

Hypertension is the most important and strongest modifiable risk factor of CVD. ${ }^{5,6}$ Two hundred million Chinese are estimated to be hypertensive, which means that 1 in 5 adults was afflicted by hypertension in 2010. ${ }^{2}$ Numerous population-based studies have investigated the significance of the resting heart rate to predict cardiovascular morbidity and mortality in the general population, $, 7,8$ elderly subjects ${ }^{9,10}$ and patients with CVDs. ${ }^{11-13}$ Previous studies have demonstrated that heart rate is a strong correlate of blood pressure (BP), and is not only faster in the individuals with high BP but is also a predictor of hypertension. ${ }^{14-16}$ However, no studies have specifically evaluated the combined effects of heart rate and hypertension on the risk of stroke and coronary heart disease (CHD) incidence in an Inner Mongolian population. The purpose of this study was to prospectively evaluate the combined effects of hypertension and heart rate on the risk of stroke and CHD on the basis of an $~ 10$-year follow-up study in an Inner Mongolian population of China.

\section{MATERIALS AND METHODS}

Study participants

A cross-sectional study was conducted between 2002 and 2003 in Inner Mongolia, an autonomous region in north China. Based on the cross-sectional

${ }^{1}$ Department of Epidemiology, School of Public Health, Medical College of Soochow University, Suzhou, China; ${ }^{2}$ Jiangsu Key Laboratory of Preventive and Translational Medicine for Geriatric Diseases, Soochow University, Suzhou, China; ${ }^{3}$ School for Radiological and Interdisciplinary Sciences (RAD-X), and School of Radiation Medicine and Protection, Soochow University, Suzhou, China and ${ }^{4}$ Department of Cardiology, the First Affiliated Hospital of China Medical University, Shenyang, China

${ }^{5}$ These authors contributed equally to this work.

Correspondence: Dr Y Zhang, Department of Epidemiology, School of Public Health, Medical College of Soochow University, 199 Renai Road, Industrial Park District, Suzhou 215123, China. 
study, a prospective cohort study was conducted from June 2003 to July 2012. Detailed descriptions of methods for study participant recruitment and baseline data collection have previously been described. ${ }^{17}$ Briefly, study participants $\geqslant 20$ years of age were recruited from 32 villages in 2 adjacent townships located in Kezuohou Banner (county) and Naiman Banner in Inner Mongolia. Nearly all local residents are Mongolians and have lived there for a long time from generation to generation; they have maintained traditional manners and customs of Mongolian ethnicity, and their diets are high in fat and salt. A total of 3475 Mongolian people $\geqslant 20$ years of age lived in these villages. Among them, 889 people were excluded because they refused to participate or had CVDs or endocrine diseases, including hyper/hypothyroidism. Finally, a total of 2589 individuals were included in this study. This study was approved by the Soochow University Ethics Committee. Written informed consent was obtained from all of the study participants.

\section{Data collection}

The data on the demographic characteristics, lifestyle risk factors, family history of CVDs and medical history were obtained using a standard questionnaire administered by trained staff. Cigarette smoking was defined as having smoked at least one cigarette per day for 1 year or more. The information regarding amount and type of alcohol consumed during the past years was collected, and alcohol drinking was defined as consuming at least $50 \mathrm{~g}$ of distillate spirits ( $\sim 50 \%$ alcohol concentrate, namely, $25 \mathrm{~g}$ of alcohol) per day for 1 year or more.

Three BP measurements were taken for each participant while participants were seated, using a mercury sphygmomanometer according to a standard protocol. ${ }^{18}$ The first and fifth Korotkoff sounds were recorded as systolic and diastolic BP, respectively. The mean of these three BP measurements was used for data analysis. Hypertension was defined as systolic BP $\geqslant 140 \mathrm{~mm} \mathrm{Hg}$ and/or diastolic BP $\geqslant 90 \mathrm{~mm} \mathrm{Hg}$ and/or use of antihypertensive medication in the recent 2 weeks. Resting heart rates were measured at baseline by a stethoscope at the apex of the heart and counted for a 60-s interval. Heart rate was grouped comparing the upper tertile ( $\geqslant 80$ b.p.m.) to the bottom 2 tertiles ( $<80$ b.p.m.). $\mathrm{BP}$ and heart rate were only measured at baseline. Body weight and height were measured with subjects wearing light clothing and without shoes by trained staff. The body mass index (BMI) was calculated as weight in kilograms divided by the square of the height in meters. The waist circumference (WC) was measured at the level of $1 \mathrm{~cm}$ above the umbilicus.

Fasting blood samples were collected during the morning after at least $8 \mathrm{~h}$ of fasting for all of the participants. Plasma and serum were frozen at $-80^{\circ} \mathrm{C}$ until testing. A modified hexokinase enzymatic method was applied to test plasma glucose levels. Hyperglycemia was defined as fasting plasma glucose $\geqslant 6.1 \mathrm{mmoll}^{-1}$. Total cholesterol, high-density lipoprotein cholesterol, and triglycerides were enzymatically analyzed using a Beckman Synchron CX5 Delta Clinical System (Beckman Coulter, Fullerton, CA, USA) with commercial reagents. Low-density lipoprotein cholesterol levels were calculated using the Friedewald equation for participants with $<400 \mathrm{mg} \mathrm{dl}^{-1}$ triglycerides. ${ }^{19}$

\section{Follow-up and outcome assessment}

All of the participants were followed from June 2003 to July 2012. Study outcomes were the development of CVD consisting of stroke and CHD. Stroke was defined as 'evidence of an acute disturbance of focal areas in the brain lasting for $\geqslant 24 \mathrm{~h}$ and thought to be due to intracranial hemorrhage or ischemia, ${ }^{20}$ and was confirmed by computed tomography and magnetic resonance imaging scan. CHD was diagnosed based on symptoms, electrocardiograms changes, cardiac enzymes and autopsy findings according to the criteria of the World Health Organization MONICA project. ${ }^{21}$ The participants who did not experience a stroke or CHD, who died from other causes or who were lost to follow-up were defined as censored. If the participant was contacted and found to have experienced a stroke or CHD, the date of incidence was defined as the end-point date. The data were censored at the time of the contact if the participant was reached and was found not to have had a CVD and at the day we contacted the participant the last time if he/she was lost to follow-up. For those who died from other causes, the data were censored at the time of death date in the medical records. Four county hospitals with modern diagnostic facilities provide the residents of the 32 villages with medical services. Since 2004, household surveys of all participants were conducted every
2 years to determine new CVD events. Trained staff interviewed either the participants or their relatives (if participants were dead or unable to communicate) and completed a medical status questionnaire. If a participant reported that a stroke or CHD occurred during the period since the last survey, the staff reviewed hospital records, including outpatient or admission records, the discharge summary and especially electrocardiogram, cardiac enzyme, autopsy, computed tomography and magnetic resonance imaging scan results, to confirm.

\section{Statistical analysis}

According to the hypertensive status and heart rate, the participants were divided into four groups: no hypertension with a heart rate $<80$ b.p.m., no hypertension with a heart rate $\geqslant 80$ b.p.m., hypertension with a heart rate $<80$ b.p.m. and hypertension with a heart rate $\geqslant 80$ b.p.m. The baseline characteristics of continuous variables were compared in four subgroups using analysis of variance. The categorical variables were expressed as a percentage and compared between the groups using a $\chi^{2}$ test. The log-rank test was used to compare cumulative risk of events among the four subgroups. Multivariate Cox proportional hazard models were used to compute the hazard ratios (HRs) and 95\% confidence intervals of stroke or CHD incidence across the four subgroups, adjusting for age, gender, BMI, smoking and drinking status, WC, family history of CVD, hyperglycemia and lipid levels. We also set a multiplicative interact term of hypertension and heart rate in the Cox proportional hazards model and tested its effects on stroke or CHD incidence independent of hypertension, heart rate and other confounding factors. In addition, we assessed the discriminatory value of hypertension/heart rate $\geqslant 80$ b.p.m. by computing the area under receiver operating characteristic curves (AUC) and comparing a model including hypertension and heart rate $\geqslant 80$ b.p.m. and other conventional risk factors with a model including only other conventional risk factors. All of the $P$ values were two-tailed, and a significance level of 0.05 was used. Statistical analysis was conducted using SAS statistical software (version 9.1, Cary, NC, USA) and R statistical software (version 2.15, Vienna, Austria).

\section{RESULTS}

As of 31 July 2012, we have followed-up the participants for an average of 9.2 years. Among 2589 participants, 6 were lost to follow-up, and the follow-up rate was $99.8 \%$.

Fifty-nine participants were excluded for missing key variables, and a total of 2530 people were included in the final analysis. During the follow-up period, 195 participants experienced CVD, including 120 stroke and $75 \mathrm{CHD}$ events. The cumulative incidence rates of stroke and $\mathrm{CHD}$ were $4.74 \%$ and $2.96 \%$, respectively; the incidence densities were 513 and 321 per 100000 person-years, respectively.

\section{Demographic and clinical characteristics}

Table 1 summarizes the demographic and clinical characteristics of 2530 participants according to hypertensive status and heart rate. Conventional risk factors, such as age, gender, smoking and drinking status, family history of CVD, triglycerides, total cholesterol, lowdensity lipoprotein cholesterol, fast plasma glucose, hyperglycemia, WC and BMI, were significantly different among the four subgroups. Namely, hypertensive participants either in a heart rate $<80$ b.p.m. group or in a heart rate $\geqslant 80$ b.p.m. group tended to be older, men, drinkers, had a higher rate of family history of CVD, and higher levels of triglycerides, total cholesterol, low-density lipoprotein cholesterol, WC and BMI. Hypertensive and non-hypertensive participants with a heart rate $\geqslant 80$ b.p.m. were likely to have higher rates of hyperglycemia, lower rates of smoking and higher levels of fast plasma glucose.

\section{Combined effect on the risk of stroke}

During the 9.2 years of follow-up, the cumulative incidence rates of stroke among the normotensives with a heart rate $<80$ b.p.m., 
Table 1 Demographic and clinical characteristics of 2530 participants according to hypertensive status and heart rate in Inner Mongolia, China

\begin{tabular}{|c|c|c|c|c|c|}
\hline & $\begin{array}{c}\text { Normotensives/heart } \\
\text { rate }<80 \text { b.p.m. }\end{array}$ & $\begin{array}{c}\text { Normotensives/heart } \\
\text { rate } \geqslant 80 \text { b.p.m. }\end{array}$ & $\begin{array}{c}\text { Hypertensives/heart } \\
\text { rate }<80 \text { b.p.m. }\end{array}$ & $\begin{array}{c}\text { Hypertensives/heart } \\
\text { rate } \geqslant 80 \text { b.p.m. }\end{array}$ & P-value \\
\hline Number of participants & 1058 & 530 & 552 & 390 & \\
\hline Age (years) & $43.44 \pm 11.20$ & $42.68 \pm 11.81$ & $52.03 \pm 11.95$ & $51.64 \pm 11.93$ & $<0.001$ \\
\hline Male (\%) & 42.25 & 21.51 & 54.17 & 43.85 & $<0.001$ \\
\hline Cigarette smoking (\%) & 45.27 & 35.66 & 50.54 & 44.36 & $<0.001$ \\
\hline Alcohol drinking (\%) & 30.43 & 19.43 & 46.56 & 41.03 & $<0.001$ \\
\hline Family history of CVD (\%) & 6.43 & 5.47 & 23.55 & 26.67 & $<0.001$ \\
\hline $\mathrm{TG}\left(\mathrm{mmol} \mathrm{I}^{-1}\right)$ & $1.09 \pm 0.95$ & $1.07 \pm 0.77$ & $1.39 \pm 1.11$ & $1.79 \pm 2.28$ & $<0.001$ \\
\hline $\mathrm{TC}\left(\mathrm{mmol} \mathrm{I}^{-1}\right)$ & $3.61 \pm 1.05$ & $3.56 \pm 1.03$ & $3.99 \pm 1.14$ & $3.97 \pm 1.32$ & $<0.001$ \\
\hline LDL cholesterol (mmol I-1) & $2.21 \pm 0.98$ & $2.19 \pm 0.95$ & $2.53 \pm 1.05$ & $2.45 \pm 1.18$ & $<0.001$ \\
\hline HDL cholesterol (mmoll-1) & $1.18 \pm 0.32$ & $1.17 \pm 0.34$ & $1.18 \pm 0.33$ & $1.16 \pm 0.34$ & 0.718 \\
\hline FPG $\left(\mathrm{mmol} \mathrm{I}^{-1}\right)$ & $4.72 \pm 0.90$ & $5.04 \pm 1.20$ & $5.02 \pm 1.05$ & $5.56 \pm 1.83$ & $<0.001$ \\
\hline Hyperglycemia (\%) & 6.14 & 13.02 & 10.14 & 21.79 & $<0.001$ \\
\hline WC $(\mathrm{cm})$ & $79.43 \pm 8.42$ & $77.67 \pm 8.84$ & $84.23 \pm 9.90$ & $83.72 \pm 10.55$ & $<0.001$ \\
\hline $\mathrm{BMI}\left(\mathrm{kg} \mathrm{m}^{-2}\right)$ & $22.01 \pm 3.10$ & $21.32 \pm 3.29$ & $23.17 \pm 3.61$ & $22.89 \pm 3.96$ & $<0.001$ \\
\hline
\end{tabular}

Abbreviations: BMI, body mass index; CVD, cardiovascular disease; FPG, fast plasma glucose; HDL, high-density lipoprotein; LDL, low-density lipoprotein; TC, total cholesterol; TG, triglycerides; WC, waist circumference.

Table 2 Multivariable adjusted HRs of heart rate and hypertension for the development of stroke and coronary heart disease

\begin{tabular}{|c|c|c|c|c|}
\hline & \multirow[b]{2}{*}{ Cases (\%) } & \multicolumn{3}{|c|}{ Multivariable adjusted } \\
\hline & & $H R$ & $95 \% \mathrm{Cl}$ & P-value \\
\hline \multicolumn{5}{|l|}{ Stroke } \\
\hline Heart rate (per b.p.m.) ${ }^{a}$ & $120(4.74)$ & 1.01 & $1.00-1.02$ & 0.221 \\
\hline Heart rate $\geqslant 80$ b.p.m. ${ }^{a}$ & $48(5.22)$ & 1.05 & $0.71-1.55$ & 0.819 \\
\hline Hypertension ${ }^{b}$ & $91(9.66)$ & 2.69 & $1.70-4.25$ & $<0.001$ \\
\hline \multicolumn{5}{|l|}{ Coronary heart disease } \\
\hline Heart rate (per b.p.m.) & $75(2.96)$ & 1.01 & $1.00-1.03$ & 0.119 \\
\hline Heart rate $\geqslant 80$ b.p.m. ${ }^{a}$ & $35(3.80)$ & 1.19 & $0.74-1.93$ & 0.473 \\
\hline Hypertension ${ }^{b}$ & $49(5.20)$ & 2.33 & $1.36-4.00$ & 0.002 \\
\hline \multicolumn{5}{|l|}{ Cardiovascular disease } \\
\hline Heart rate (per b.p.m.) & $195(7.71)$ & 1.01 & $1.00-1.02$ & 0.044 \\
\hline Heart rate $\geqslant 80$ b.p.m. ${ }^{a}$ & $83(9.02)$ & 1.35 & $1.01-1.82$ & 0.046 \\
\hline Hypertension ${ }^{\mathrm{b}}$ & $140(14.86)$ & 2.48 & $1.75-3.50$ & $<0.001$ \\
\hline
\end{tabular}

Abbreviations: $\mathrm{Cl}$, confidential interval; $\mathrm{HR}$, hazard ratio.

aAdjusted for age, sex, BMI, smoking and drinking status, WC, family history of CVD, systolic blood pressure, diastolic blood pressure, blood glucose and lipids.

${ }^{b}$ Adjusted for age, sex, BMI, smoking and drinking status, WC, family history of CVD, heart rate, blood glucose and lipids.

normotensives with a heart rate $\geqslant 80$ b.p.m., hypertensives with a heart rate $<80$ b.p.m. and hypertensives with a heart rate $\geqslant 80$ b.p.m. were $1.61 \%, 2.26 \%, 9.23 \%$ and $9.96 \%$, respectively (log-rank $P<0.001$ ). The adjusted HRs of stroke for heart rate and hypertension are shown in Table 2. Hypertension was significantly associated with stroke but heart rate was not, no matter as a continuous variable or categorical variable. As shown in Table 3, compared with the normotensives with a heart rate $<80$ b.p.m., the multivariable adjusted HRs of stroke for hypertensives with a heart rate $<80$ b.p.m. and hypertensives with a heart rate $\geqslant 80$ b.p.m. were statistically significant (all $P<0.001$ ). The hypertensives with a heart rate $\geqslant 80$ b.p.m. had the highest risk of stroke. No significant interaction was detected between hypertension and heart rate on the development of stroke $(P=0.29)$. The AUC for the model including hypertension and heart rate $\geqslant 80$ b.p.m., and other conventional risk factors was significantly larger than that for the model only including other conventional factors (0.836 vs. 0.854 , $P=0.023$; Figure 1a).

\section{Combined effect on the risk of CHD}

The cumulative incidence rates of $\mathrm{CHD}$ among the four subgroups were $1.61 \%, 1.70 \%, 4.17 \%$ and $6.67 \%$, respectively (log-rank $P<0.001)$. Hypertension was associated with $\mathrm{CHD}$, whereas heart rate was not a significant independent factor for $\mathrm{CHD}$, regardless of whether it was designated as a continuous variable or categorical variable. Compared with the normotensives with a heart rate $<80$ b.p.m., the multivariable adjusted HRs of CHD for hypertensives with a heart rate $<80$ b.p.m. and hypertensives with a heart rate $\geqslant 80$ b.p.m. were statistically significant (all $P<0.001$ ). The hypertensives with a heart rate $\geqslant 80$ b.p.m. were at the highest risk of $\mathrm{CHD}$. No significant interaction was detected between hypertension and heart rate on the development of CHD $(P=0.59)$. The AUC for the model including hypertension and a heart rate $\geqslant 80$ b.p.m., and other conventional risk factors was larger than for the model including only other conventional risk factors ( 0.730 vs. $0.665, P=0.003$; Figure $1 \mathrm{~b})$.

\section{Combined effect on the risk of CVD}

The cumulative incidence rates of CVD among the four subgroups were $3.21 \%, 3.96 \%, 14.13 \%$ and $15.90 \%$, respectively (log-rank $P<0.001)$. Hypertension and heart rate were both associated with the development of CVD. Compared with the normotensives with a heart rate < 80 b.p.m., the multivariable adjusted HRs of CVD for hypertensives with a heart rate $<80$ b.p.m. and hypertensives with a heart rate $\geqslant 80$ b.p.m. were statistically significant (all $P<0.001$ ). The hypertensives with a heart rate $\geqslant 80$ b.p.m. were at the highest risk of CVD. No significant interaction was detected between hypertension and heart rate on the development of CVD $(P=0.56)$. The AUC for the model including hypertension and heart rate $\geqslant 80$ b.p.m., and other conventional risk factors was larger than for the model including only other conventional risk factors (0.806 vs. $0.789, P=0.033$; Figure 1c). 
Table 3 Combined effects of hypertension and heart rate on the development of stroke and coronary heart disease

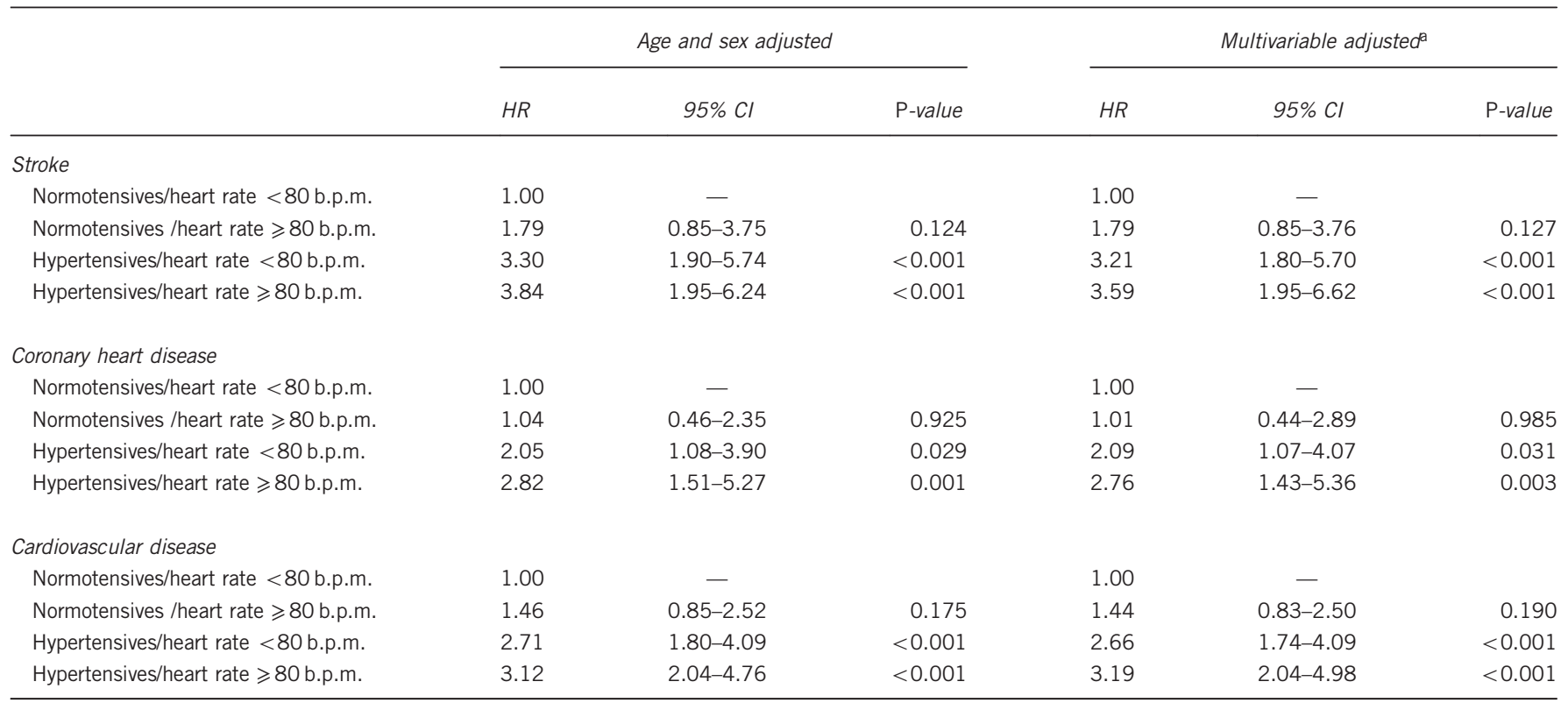

Abbreviations: $\mathrm{Cl}$, confidence interval; $\mathrm{HR}$, hazard ratio.

aAdjusted for age, sex, BMI, smoking and drinking status, WC, family history of CVD, hyperglycemia and lipids.

a

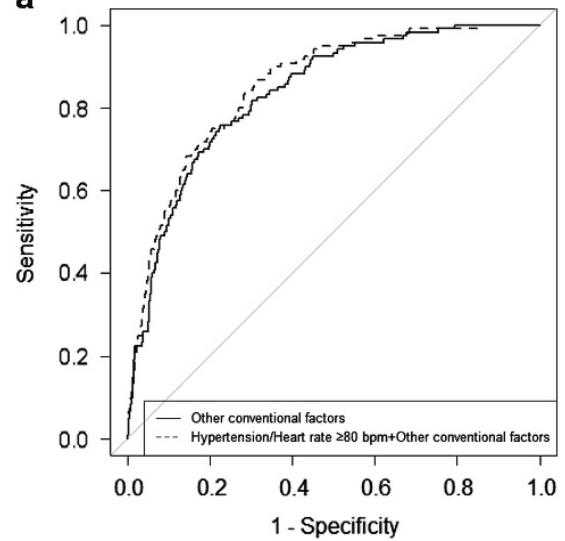

b

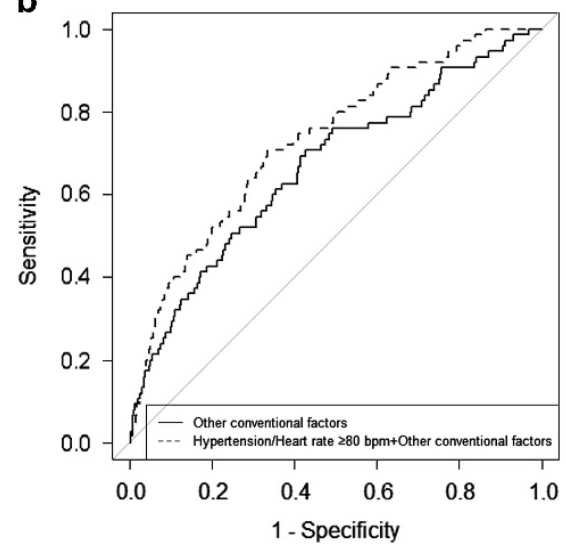

C

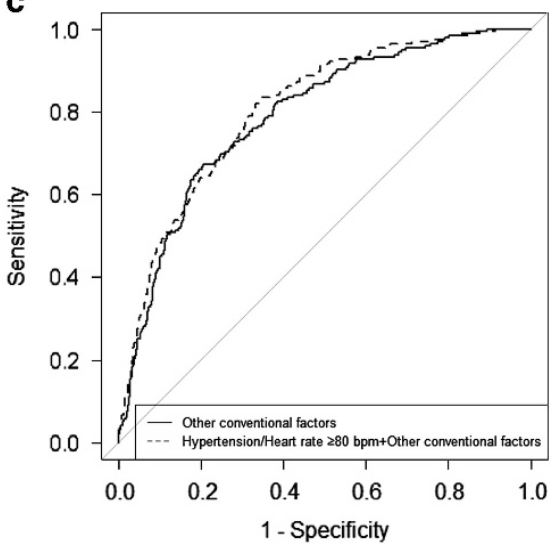

Figure 1 Area under the curve for the prediction of stroke (a), coronary heart disease (CHD) (b), and cardiovascular disease (CVD) (c), incidence according to hypertension/heart rate $\geqslant 80$ b.p.m. and other conventional factors. Other conventional factors include age, sex, body mass index, smoking and status, waist circumference, family history of CVD, hyperglycemia and lipids.

\section{DISCUSSION}

In this population-based prospective cohort study of an Inner Mongolian population, high heart rate was an independent risk factor of CVD (combined outcome of CHD and Stroke). The participants with hypertension and a heart rate $<80$ b.p.m. or with hypertension and a heart rate $\geqslant 80$ b.p.m. had a significantly higher risk for both stroke and CHD compared to those with no hypertension and a heart rate $<80$ b.p.m. Hypertensives with a heart rate $\geqslant 80$ b.p.m. had the highest risk of CHD or stroke in this population. Our study is the first to evaluate the combined effects of heart rate and hypertension on the risk of stroke and CHD in an Inner Mongolian population of China.

Hypertension is an important public health challenge worldwide because of its high frequency and concomitant risk of CVD. ${ }^{6,22,23}$ More than a quarter of the world's adult population, namely nearly one billion people, had hypertension in year 2000, and this proportion will increase to $29 \%-1.56$ billion — by the year $2025 .{ }^{6}$ Clinical trials have documented that lowering BP could reduce the risk of CVD (including stroke and CHD) in hypertensives. ${ }^{24,25}$ In our study, $\sim 37.2 \%$ of the participants had hypertension, which was higher than the proportion worldwide, and hypertension was an independent risk factor for stroke and CHD. Thus, effective control and management of BP should be considered to be an important strategy to reduce the risk of CVD in this population. Recently, some epidemiological studies have investigated the association between high heart rate and CVD, which showed that high heart rate was a major risk factor for atherosclerosis and CVD as well as total mortality. ${ }^{14,26-28}$ Our results support the association of heart rate as an independent risk factor of CVD, although there was no significant association between heart rate and stroke or CHD.

Previous studies have demonstrated that individuals with high BP typically have faster heart rates compared with those with normal BP. ${ }^{14}$ The results from the Systolic Hypertension in Europe Trial showed that in untreated older patients with isolated systolic hypertension, a clinic heart rate $>79$ b.p.m. was a significant predictor 
of cardiovascular and noncardiovascular mortality. ${ }^{29}$ Thus, it appears that a high heart rate and hypertension may act synergistically in the development of CVD. ${ }^{30}$ However, no studies have specifically evaluated the combined effects of heart rate and hypertension on the risk of stroke and CHD incidence in an Inner Mongolian population. Our findings showed that hypertensives with a heart rate $\geqslant 80$ b.p.m. had the highest risk of stroke, CHD and CVD among the four subgroups, with a 3.59-, 2.76- and 3.19-fold increased risk, respectively, compared with normotensives with a heart rate $<80$ b.p.m., which indicated that the coexistence of hypertension and high heart rate may be valuable predictor of stroke and $\mathrm{CHD}$ incidence.

High heart rate is related to increased sympathetic activity, which reflects increased stress or anxiety and may lead to atherosclerosis and increased cardiovascular morbidity and mortality. ${ }^{31}$ The combination of hypertension and high heart rate may induce atherosclerotic lesions and the development of stroke and CHD. Experimental and clinical studies have already suggested that heart rate reduction may improve coronary endothelial function, slow atherosclerosis and reduce the stroke volume. ${ }^{32-34}$ However, hypertension itself is one of the most important risk factors for stroke and CHD. In addition, a high heart rate increases cardiac output, aggravates vasoconstriction and elevating BP. ${ }^{35}$ Furthermore, both atrial fibrillation related to a high heart rate and BP elevation are important risk factors of stroke and congestive heart failure related to $\mathrm{CHD}$. Thus, reducing the heart rate may have an additional preventive effect on stroke and CHD based on the administration of antihypertensive treatment in hypertensives.

This cohort study was conducted in a Chinese Mongolian population who had a high prevalence of hypertension and different living customs from Chinese Han people. The study participants were homogeneous regarding their genetic background and environmental exposures. In addition, this cohort had a low rate of loss to follow-up and a relatively long follow-up time. These stronger points were helpful for us to obtain a less biased association between exposure variables and outcome events. However, there were two potential limitations in this study. First, the sample size of our study is comparatively small, and the relatively low number of stroke and CHD events was observed, which might underestimate the association between heart rate and stroke and $\mathrm{CHD}$ because of relatively low power. Second, BP and heart rate were recorded only once at baseline, and we have no data on the possible changes in $\mathrm{BP}$ and heart rate during follow-up. Future studies should examine the association between $\mathrm{BP}$ and heart rate change and the risk of stroke and CHD. In addition, the clinic heart rate may be influenced by environmental stimuli such as the so-called white-coat effect. ${ }^{36}$ Studies have shown that the home-measured resting heart rate could avoid the white-coat effect and has better reproducibility. ${ }^{37}$ In our study, the resting heart rate was determined mostly in villagers' homes, which might reduce the impact of environmental stimuli. Finally, 889 people of these 2 adjacent townships did not participate in this cohort, which might have introduced some selection bias. We believe that this bias is minimal because it is unlikely that the individuals chose not to participate due to their $\mathrm{BP}$ and heart rate.

In conclusion, a high heart rate was an independent risk factor of CVD. Hypertensives with a high heart rate had the highest risk of stroke and CHD in the Inner Mongolians. These results indicate that the coexistence of hypertension and a high heart rate may be valuable predictor of stroke and $\mathrm{CHD}$, and further preventive strategies are needed to simultaneously target hypertension and high heart rate.

\section{CONFLICT OF INTEREST}

The authors declare no conflict of interest.

\section{ACKNOWLEDGEMENTS}

We thank the study participants, the Kezuohouqi Banner Center for Disease Prevention and Control and the Naiman Banner Center for Disease Prevention and Control for their support and assistance. This study was supported by the National Natural Science Foundation of China (Grant nos. 81172761 and 30972531) and a Project of the Priority Academic Program Development of Jiangsu Higher Education Institutions.

1 Narayan KV, Ali MK, Koplan JP. Global noncommunicable diseases-where worlds meet. N Engl J Med 2010; 363: 1196-1198.

2 Hu SS, Kong LZ, Gao RL, Zhu ML, Wang W, Wang YJ, Wu ZS, Chen WW, Liu MB. Outline of the report on cardiovascular disease in china, 2010. Biomed Environ Sci 2012; 25: 251-256.

3 Gaziano T. Economic burden and the cost-effectiveness of treatment of cardiovascular diseases in Africa. Heart 2008; 94: 140-144.

4 Deaton C, Froelicher ES, Wu LH, Ho C, Shishani K, Jaarsma T. The global burden of cardiovascular disease. Eur J Cardiovasc Nurs 2011; 10: S5-S13.

5 Collaboration PS. Age-specific relevance of usual blood pressure to vascular mortality: a meta-analysis of individual data for one million adults in 61 prospective studies. Lancet 2002; 360: 1903-1913.

6 Kearney PM, Whelton M, Reynolds K, Muntner P, Whelton PK, He J. Global burden of hypertension: analysis of worldwide data. Lancet 2005; 365: 217-223.

7 Cooney MT, Vartiainen E, Laakitainen T, Juolevi A, Dudina A, Graham IM. Elevated resting heart rate is an independent risk factor for cardiovascular disease in healthy men and women. Am Heart J 2010; 159: 612-619. e613.

8 Fujiura Y, Adachi H, Tsuruta M, Jacobs DR Jr, Hirai Y, Imaizumi T. Heart rate and mortality in a japanese general population:: an18-year follow-up study. J Clin Epidemiol 2001; 54: 495-500.

9 Palatini P, Casiglia E, Julius S, Pessina AC. High heart rate: a risk factor for cardiovascular death in elderly men. Arch Intern Med 1999; 159: 585-592.

10 Palatini P, Thijs L, Staessen JA, Fagard RH, Bulpitt CJ, Clement DL, de Leeuw PW, Jaaskivi M, Leonetti G, Nachev C. Predictive value of clinic and ambulatory heart rate for mortality in elderly subjects with systolic hypertension. Arch Intern Med 2002; 162: 2313-2321.

11 Lonn EM, Rambihar S, Gao P, Custodis FF, Sliwa K, Teo KK, Yusuf S, Böhm M. Heart rate is associated with increased risk of major cardiovascular events, cardiovascular and all-cause death in patients with stable chronic cardiovascular disease: an analysis of ontarget/transcend. Clin Res Cardiol 2014; 103: 149-159.

12 Böhm M, Cotton D, Foster L, Custodis F, Laufs U, Sacco R, Bath PM, Yusuf S, Diener H-C. Impact of resting heart rate on mortality, disability and cognitive decline in patients after ischaemic stroke. Eur Heart J 2012; 33: 2804-2812.

13 Bemelmans RH, van der Graaf Y, Nathoe HM, Wassink AM, Vernooij JW, Spiering W, Visseren FL. The risk of resting heart rate on vascular events and mortality in vascular patients. Int J Cardiol 2013; 168: 1410-1415.

14 Kolloch $R$, Legler UF, Champion A, Cooper-DeHoff RM, Handberg $E$, Zhou $Q$ Pepine CJ. Impact of resting heart rate on outcomes in hypertensive patients with coronary artery disease: Findings from the international verapamil-sr/trandolapril study (invest). Eur Heart J 2008; 29: 1327-1334.

15 Morcet J-F, Safar M, Thomas F, Guize L, Benetos A. Associations between heart rate and other risk factors in a large French population. J Hypertens 1999; 17: 1671-1676.

16 Benetos A, Rudnichi A, Thomas F, Safar M, Guize L. Influence of heart rate on mortality in a French population role of age, gender, and blood pressure. Hypertension 1999; 33: 44-52.

$17 \mathrm{Li} \mathrm{H}$, Xu T, Tong W, Liu Y, Zhao L, Zhang Y. Comparison of cardiovascular risk factors between prehypertension and hypertension in a Mongolian population, Inner Mongolia, China. Circ J 2008; 72: 1666-1673.

18 Perloff D, Grim C, Flack J, Frohlich ED, Hill M, McDonald M, Morgenstern BZ. Human blood pressure determination by sphygmomanometry. Circulation 1993; 88: 2460-2470.

19 Friedewald WT, Levy RI, Fredrickson DS. Estimation of the concentration of low-density lipoprotein cholesterol in plasma, without use of the preparative ultracentrifuge. Clin Chem 1972; 18: 499-502.

20 Goldstein M, Barnett H, Orgogozo J, Sartorius N, Symon L, Vereshchagin N. Stroke-1989: Recommendations on stroke prevention, diagnosis, and therapy. Report of the who task force on stroke and other cerebrovascular disorders. Stroke 1989; 20: 1407-1431.

21 Tuomilehto J, Kuulasmaa K. Who monica project: assessing chd mortality and morbidity. Int J Epidemiol 1988; 18: S38-S45.

22 He J, Whelton PK. Epidemiology and prevention of hypertension. Med Clin North Am 1997; 81: 1077-1097.

23 He J, Gu D, Wu X, Reynolds K, Duan X, Yao C, Wang J, Chen C-S, Chen J, Wildman RP. Major causes of death among men and women in china. N Engl J Med 2005; 353: 1124-1134.

24 Law M, Morris J, Wald N. Use of blood pressure lowering drugs in the prevention of cardiovascular disease: Meta-analysis of 147 randomised trials in the context of expectations from prospective epidemiological studies. Brit Med J 2009; 338: b1665. 
25 Trialists'Collaboration BPLT. Effects of different blood-pressure-lowering regimens on major cardiovascular events: Results of prospectively-designed overviews of randomised trials. Lancet 2003; 362: 1527-1535.

26 Palatini P. Elevated heart rate: A "new" cardiovascular risk factor? Progr Cardiovasc Dis 2009; 52: 1-5.

27 Jensen MT, Marott JL, Jensen GB. Elevated resting heart rate is associated with greater risk of cardiovascular and all-cause mortality in current and former smokers. Int J Cardiol 2011; 151: 148-154.

28 Fox K, Ford I, Steg PG, Tendera M, Robertson M, Ferrari R. Heart rate as a prognostic risk factor in patients with coronary artery disease and left-ventricular systolic dysfunction (beautiful): a subgroup analysis of a randomised controlled trial. Lancet 2008; 372: 817-821.

29 Palatini P, Thijs L, Staessen JA, Fagard RH, Bulpitt CJ, Clement DL, de Leeuw PW, Jaaskivi M, Leonetti G, Nachev C, O'Brien ET, Parati G, Rodicio JL, Roman E, Sarti C, Tuomilehto J. Predictive value of clinic and ambulatory heart rate for mortality in elderly subjects with systolic hypertension. Arch Intern Med 2002; 162 2313-2321.

30 Gillman MW, Kannel WB, Belanger A, D'Agostino RB. Influence of heart rate on mortality among persons with hypertension: The framingham study. Am Heart J 1993; 125: $1148-1154$
31 Palatini P. Sympathetic overactivity in hypertension: a risk factor for cardiovascular disease. Curr Hypertens Rep 2001; 3: S3-S9.

32 Custodis F, Gertz K, Balkaya M, Prinz V, Mathar I, Stamm C, Kronenberg G, Kazakov A, Freichel M, Böhm M. Heart rate contributes to the vascular effects of chronic mental stress effects on endothelial function and ischemic brain injury in mice. Stroke 2011 42: 1742-1749.

33 Bassiouny HS, Zarins CK, Lee DC, Skelly CL, Fortunato JE, Glagov S. Diurnal heart rate reactivity: A predictor of severity of experimental coronary and carotid atherosclerosis. J Cardiovasc Risk 2002; 9: 331-338.

34 Inoue T, Iseki K, Ohya Y. Heart rate as a possible therapeutic guide for the prevention of cardiovascular disease. Hypertens Res 2013; 36: 838-844.

35 Elstad M, Walløe L, Chon KH, Toska K. Low-frequency fluctuations in heart rate, cardiac output and mean arterial pressure in humans: What are the physiological relationships? J Hypertens 2011; 29: 1327-1336.

36 Palatini P, Julius S. Heart rate and the cardiovascular risk. J Hypertens 1997; 15: 3-17.

37 Ushigome E, Fukui M, Hamaguchi M, Tanaka T, Atsuta H, Ohnishi M, Tsunoda S, Yamazaki M, Hasegawa G, Nakamura N. Home-measured heart rate is associated with albuminuria in patients with type 2 diabetes mellitus: a post-hoc analysis of a cross-sectional multicenter study. Hypertens Res 2014; 37: 533-537. 